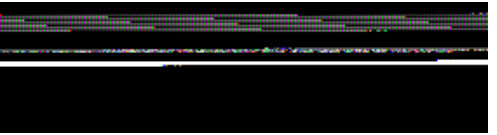

This information is current as of April 25, 2023.
Identification of Chronic Active Multiple Sclerosis Lesions on 3T MRI

M. Absinta, P. Sati, A. Fechner, M.K. Schindler, G. Nair and D.S. Reich

AJNR Am J Neuroradiol 2018, 39 (7) 1233-1238

doi: https://doi.org/10.3174/ajnr.A5660

http://www.ajnr.org/content/39/7/1233 


\title{
Identification of Chronic Active Multiple Sclerosis Lesions on 3T MRI
}

\author{
(D) M. Absinta, (DP. Sati, (D)A. Fechner, (D) M.K. Schindler, (D) G. Nair, and DD.S. Reich
}

\begin{abstract}
BACKGROUND AND PURPOSE: MR imaging-pathologic studies have reported that paramagnetic rims on 7T susceptibility-based MR imaging identify, in vivo, the subset of MS lesions with compartmentalized inflammation at the lesion edge and associated remyelination failure. Here, we assessed the reliability of detecting these rims on high-resolution 3T phase images.
\end{abstract}

MATERIALS AND METHODS: High-resolution T2* and phase MR imaging was collected in 20 patients with MS at 3T (3D segmented EPI, $0.65 \mathrm{~mm}^{3}$ ) and 7T (2D gradient-echo, $0.2 \times 0.2 \times 1 \mathrm{~mm}$ ) MR imaging. In each case, 5 discrete chronic (nonenhancing) MS lesions were selected on T2 FLAIR images for rim evaluation. Five raters experienced in MS imaging contributed to the rim assessment, of whom 3 worked independently on 3T data, and 2, on 7T data. Consensus agreement was reached for both 3T and 7T rim evaluations. Discrepancies between 3T and 7T were discussed, and consensus was reached.

RESULTS: Phase rims were seen in 34 lesions at 7T and in 36 lesions at 3T by consensus. Inter- and intrarater reliability were "substantial/ good" both at 3T and 7T analysis (Cohen $\kappa,>0.71$ ). Based on consensus agreement, the reliability of rim visualization at 3T versus $7 \mathrm{~T}$ was 0.78 $(\kappa)$ with a pair-wise agreement of $90 \%$. More lesions were judged to be false-positive or false-negative at 3T than at 7T.

CONCLUSIONS: Nearly all 7T paramagnetic rims can also be seen at 3T. Imaging at 3T opens the possibility of implementing paramagnetic rims as an outcome measure in multicenter, MR imaging-based clinical trials aimed at treating perilesional persistent inflammation and its potential effects on remyelination.

ABBREVIATIONS: EDSS = Expanded Disability Status Scale; QSM = Quantitative Susceptibility Mapping

n multiple sclerosis, plaques of demyelination, the hallmark of this disease, can present simultaneously at different pathologic stages. ${ }^{1,2}$ Especially relevant from a disease perspective are 2 types of chronic plaques that can be considered opposite ends of the pathologic spectrum: lesions in which, after active inflammatory demyelination, the repair process has been relatively efficient (leading to remyelination), and lesions in which

Received December 8, 2017; accepted after revision March 13, 2018.

From the Translational Neuroradiology Section (M.A., P.S., A.F., M.K.S., G.N., D.S.R.), Division of Neuroimmunology and Neurovirology, National Institute of Neurological Disorders and Stroke, National Institutes of Health, Bethesda, Maryland; and Department of Radiology (A.F.), Fondation Ophtalmologique Adolphe de Rothschild, Paris, France.

This work was supported by the Intramural Research Program of National Institute of Neurological Disorders and Stroke.

Please address correspondence to Daniel S. Reich, MD, PhD, Translational Neuroradiology Section/National Institute of Neurological Disorders and Stroke/National Institutes of Health, 10 Center Dr, MSC 1400, Building 10, Room 5C103, Bethesda, MD 20892; e-mail: daniel.reich@nih.gov

- Indicates open access to non-subscribers at www.ajnr.org

7 Indicates article with supplemental on-line photo.

http://dx.doi.org/10.3174/ajnr.A5660 regeneration has completely failed, due, in part, to residual compartmentalized inflammation at the lesion margin (ie, chronic active/slowly expanding/smoldering lesions, henceforth denoted "chronic active").,

Chronic active lesions are pathologically characterized by a hypocellular demyelinated core and a hypercellular edge of activated microglia/macrophages related to smoldering inflammation and axonal degeneration. ${ }^{1,2}$ On MR imaging, these lesions have a characteristic paramagnetic rim on 7T susceptibility-based MR imaging sequences; the paramagnetic shift is due to the stable presence of iron-laden activated microglia/macrophages ${ }^{3-11}$ and, potentially, reactive astrocytes ${ }^{12}$ at the lesion edge. Usually, paramagnetic rims are seen on $7 \mathrm{~T}$ phase images, but a proportion can also be detected on $7 \mathrm{~T} \mathrm{T2}{ }^{\star}$ magnitude images, mostly when iron content is higher. ${ }^{4-6,13,14}$ In the context of early lesion evolution, we recently reported that paramagnetic rims in active lesions colocalize with peripheral or centripetal gadolinium enhancement ${ }^{14}$ and that persistence of these rims after restoration of the bloodbrain barrier (ie, approximately 3 months after initial demyelination) predicts failure of tissue repair and remyelination. ${ }^{10}$ There- 
Table 1: Clinical and demographic data

\begin{tabular}{lllllcl}
\hline Patient No. & Sex & Age $(\mathbf{y r})$ & Clinical Phenotype & EDSS & Disease Duration (yr) & Disease-Modifying Treatment \\
\hline 1 & Female & 60 & RR & 1.5 & 11 & Dimethyl fumarate \\
2 & Female & 66 & Progressive & 7 & 28 & None \\
3 & Male & 56 & Progressive & 6 & 24 & None \\
4 & Male & 47 & RR & 1.5 & 16 & Daclizumab \\
5 & Female & 43 & RR & 2 & 43 & None \\
6 & Female & 64 & RR & 1.5 & 9 & Interferon $\beta$-la \\
7 & Female & 55 & RR & 1.5 & 2 & Glatiramer acetate \\
8 & Male & 36 & RR & 1 & 7 & None \\
9 & Male & 61 & Progressive & 6.5 & 40 & Dimethyl fumarate \\
10 & Female & 51 & Progressive & 6.5 & 24 & Glatiramer acetate \\
11 & Female & 35 & RR & 1 & 4 & None \\
12 & Female & 36 & RR & 2.5 & 18 & None \\
13 & Male & 38 & RR & 0 & 1 & None \\
14 & Female & 28 & RR & 1.5 & 1 & Mycophenolate mofetil \\
15 & Female & 33 & RR & 2 & 1 & Glatiramer acetate \\
16 & Female & 42 & RR & 2.5 & 10 & None \\
17 & Female & 25 & RR & 2 & 1 & Dimethyl fumarate \\
18 & Female & 30 & RR & 1.5 & 8 & None \\
19 & Female & 36 & RR & 1.5 & 9 & 15 \\
20 & Female & 71 & RR & 1 & & \\
\hline
\end{tabular}

Note:-RR indicates relapsing-remitting.

after, in chronic lesions, rims have been shown to be stable during short-to-medium-term follow-up (up to 3.5 years) ${ }^{13-15}$ and are not known to be affected by current disease-modifying treatments. These results establish paramagnetic rims as potential outcome measures in MR imaging-based clinical trials of therapies that might treat perilesional persistent microglial-/macrophagedriven inflammation.

Because 7T MR imaging scanners remain relatively sparse and confined to major academic centers in developed countries, the ability to reliably identify paramagnetic rims on more widespread, lower field strength (3T) scanners seems to be a necessary step toward application in multicenter clinical trials and-as important-clinical practice. A proper investigation of the detection of paramagnetic rims on 3T susceptibility-based MR images is necessary to achieve this. Here, we aimed to assess the reliability of visualizing $7 \mathrm{~T}$ paramagnetic rims (our criterion standard in vivo) on high-resolution $3 \mathrm{~T}$ susceptibility-based MR images in 20 patients with MS. At 3T, we implemented a 0.65 -mm isotropic voxel, whole-brain $3 \mathrm{D}$ segmented echo-planar imaging sequence that is already known in the MS imaging field for its benefits in assessing the central vein sign within MS lesions. $^{16-21}$

\section{MATERIALS AND METHODS \\ Participants}

Under an institutional review board-approved natural history protocol, we recruited, from May 2012 to December 2016, twenty individuals with MS ( 15 women/5 men; mean age, 46 years; range, $28-71$ years; all fulfilling the 2017 McDonald revised MS criteria ${ }^{22}$ ). Sixteen had relapsing-remitting and 4 had progressive MS. ${ }^{23}$ Experienced MS clinicians determined disability according to the Expanded Disability Status Scale (EDSS) ${ }^{24}$ and obtained clinical data. The median EDSS score was 1.5 (range, 0-7), and mean disease duration was 14 years (range, 1-40 years); clinical data were collected at the first MR imaging acquisition (Table 1 ).

\section{MR Imaging Acquisition}

Participants underwent $2 \mathrm{MR}$ imaging acquisitions approximately a year apart, one on a Magnetom Skyra (Siemens, Erlangen, Germany) 3T scanner (equipped with a body transmit coil and a 32-channel receive coil) and one on a Siemens Research System 7T MR imaging scanner (equipped with a birdcage-type transmit coil and a 32-channel receive coil). The mean time lapse between the first and second MR imaging was 0.9 years (median, 0.7 years; range, 2 days to 3.4 years). Because paramagnetic rims in chronic lesions have been shown to be stable with time, ${ }^{13-15}$ including in our cohort, we did not implement a maximum time lapse between 3T and 7T scans.

The following details the $3 \mathrm{~T}$ MR imaging protocol for rim detection:

- Whole-brain 3D segmented echo-planar imaging providing $\mathrm{T} 2{ }^{*}$ magnitude and phase contrasts $(\mathrm{TR}=64 \mathrm{~ms}$; $\mathrm{TE}=35 \mathrm{~ms}$; flip angle $=10^{\circ}$; acquisition time $=5$ minutes 46 seconds; 256 sagittal slices; $0.65-\mathrm{mm}$ isotropic voxels with a voxel size of $0.27 \mu \mathrm{L})$.

- Whole-brain 3D T2 FLAIR $(\mathrm{TR}=4800 \mathrm{~ms}$; $\mathrm{TE}=354 \mathrm{~ms}$; $\mathrm{TI}=$ $1800 \mathrm{~ms}$; flip angle $=120^{\circ}$; acquisition time $=6$ minutes $30 \mathrm{sec}$ onds; 176 sagittal slices; 1 -mm isotropic voxels with voxel size $=1$ $\mu \mathrm{L})$.

The following details the 7T MR imaging protocol for rim detection:

- $2 \mathrm{D}$ high-resolution gradient recalled-echo providing $\mathrm{T} 2^{*}$ and phase contrasts $(\mathrm{TR}=1300 \mathrm{~ms}$; $\mathrm{TE}=32 \mathrm{~ms} ; 29$ axial slices; flip angle $=50^{\circ}$; acquisition time $=8$ minutes 36 seconds; in-plane resolution $=0.2 \times 0.2 \mathrm{~mm}$; slice thickness $=1 \mathrm{~mm}$; voxel size $=0.04 \mu \mathrm{L}$ ). Three minimally overlapping slabs were acquired to allow coverage of most of the supratentorial brain.

Additional MR imaging sequences, including postcontrast T1weighted images, were typically acquired for clinical or other research purposes at both 3T and 7T MR imaging and were examined qualitatively as part of this study. 
Table 2: Intra- and interrater agreement for paramagnetic rim evaluation on $3 \mathrm{~T}$ and $7 \mathrm{~T}$ phase MRI

\begin{tabular}{lccl}
\hline \multicolumn{1}{c}{ Raters } & Percentage Agreement & Cohen $\boldsymbol{\kappa}$ & Agreement $^{28}$ \\
\hline 3T (3 raters) & & & \\
Intrarater (1 rater) & $89 \%$ & 0.77 & Substantial \\
Interrater & $86 \%$ & $0.71($ Fleiss $\kappa)$ & Substantial \\
Each rater vs 3T consensus & $86 \%-90 \%$ & $0.71-0.79$ & Substantial \\
7T (2 raters) & & & \\
Intrarater (1 rater) & $89 \%$ & 0.77 & Substantial \\
Interrater & $87 \%$ & 0.72 & Substantial \\
Each rater vs 7T consensus & $91 \%-96 \%$ & $0.81-0.91$ & Almost perfect \\
3T vs 7T & & & \\
3T vs 7T consensus & $90 \%$ & 0.78 & Substantial \\
\hline
\end{tabular}

\section{MR Imaging Analysis}

Phase postprocessing and coregistration among images were performed as previously described. ${ }^{10,14}$ Postcontrast T1-weighted images were implemented to exclude enhancing lesions from the analysis.

As previously described, ${ }^{6}$ a chronic lesion was defined as "rimpositive" when it showed a paramagnetic rim signal on phase images while being either hyper- or isointense in its inner portion. The paramagnetic rim assessment included 5 investigators with experience in imaging MS, including 2 neurologists (M.A., M.K.S.), 2 neuroradiologists (A.F., D.S.R.), and an MR physicist (P.S.). On 3T T2 FLAIR images, an investigator (M.A.) selected 5 discrete supratentorial chronic MS lesions (largest diameter of $>3$ $\mathrm{mm}$ ) from each case. The lesion location and largest lesion diameter were recorded. To remain masked to data acquired at $3 \mathrm{~T}$ and 7T, we planned rim assessment as follows:

\section{T Paramagnetic Rim Assessment}

- For each selected lesion, 3 investigators (A.F., M.K.S., D.S.R.) independently assessed the presence of paramagnetic rims on $3 \mathrm{~T}$ phase MR images. T2 FLAIR and phase images were reviewed simultaneously by each investigator using OsiriX software (http://www.osirix-viewer.com). Lesions to be analyzed were marked on MR images with a number and an arrow. A binary code was implemented as $0=$ no rim and $1=$ rim.

- After 4 months, 1 rater repeated the analysis on 3T phase data.

- Consensus agreement was subsequently reached by all investigators, and comments were recorded.

\section{T Paramagnetic Rim Assessment}

- Similar to the previous analysis, for each selected lesion, an investigator (M.A.) assessed the presence of paramagnetic rims on $7 \mathrm{~T}$ phase MR imaging.

- After 5 months, a second investigator (P.S.) performed the analysis, and the first rater repeated the analysis.

- Consensus agreement was subsequently reached, and comments were recorded.

After consensus, discrepancies between rim visualization at 3T versus $7 \mathrm{~T}$ were retrospectively investigated and discussed. The presence of rims on $3 \mathrm{~T} \mathrm{~T} 2{ }^{\star}$ magnitude images (known to be less sensitive to susceptibility shifts) was assessed retrospectively by consensus of 2 investigators (M.A., P.S.).

\section{Statistical Analysis}

Intra- and interrater reliability for rim assessment was computed on the basis of the number of raters, using the Cohen $\kappa(2$ raters) and Fleiss $\kappa$ ( 3 raters). MR imaging differences (lesion diameter and location) between lesions with-versus-without rims were assessed with a $t$ test and Fisher exact test when appropriate.

\section{RESULTS}

One hundred discrete supratentorial chronic MS lesions were initially evaluated (5 lesions per patient). Two lesions were excluded from the final analysis, one for abutting a confluent lesion and one for obvious changes in size at the second time point (time lapse, 1.7 and 1.4 years, respectively). Of the remaining 98 lesions, 35 (36\%) were periventricular; 39 (40\%), in the deep white matter; and 24 (24\%), juxtacortical/leukocortical.

\section{Lesion Rim Assessment at 3T MR Imaging (Masked to the $7 T$ Data)}

Of 98 analyzed lesions, 36 had a paramagnetic rim on 3T phase images by consensus agreement. Rim lesions were larger than nonrim lesions (mean diameter, $8.1 \pm 2.3 \mathrm{~mm}$ and $6.7 \pm 2.3 \mathrm{~mm}$, respectively; $t$ test, $P=.007)$. Overall, we did not notice any preferential lesion location: 13 lesions with rim were periventricular $(38 \%$ of total periventricular lesions), 14 were in the deep white matter (38\% of total deep white matter lesions), and 9 were juxtacortical/leukocortical (38\% of total juxtacortical/leukocortical lesions).

The intrarater and interrater agreement results are provided in Table 2 . The reliability between each rater and the final consensus agreement was "substantial/good," with a Cohen $\kappa$ ranging from 0.71 to 0.79 and a pair-wise agreement ranging from $86 \%$ to $90 \%$.

Sixteen of 36 lesions with a phase $\operatorname{rim}(44 \%)$ also showed a rim on $\mathrm{T}^{\star}$ magnitude images. The presence of a $\mathrm{T} 2{ }^{\star}$ rim was significantly associated with the detection of a rim on phase images by consensus (Fisher exact test, $P<.0001$ ).

\section{Lesion Rim Assessment at 7T MR Imaging (Masked to the 3T Data)}

Of 98 analyzed lesions, 34 had a paramagnetic rim on 7T phase images by consensus agreement. Twenty-one of 34 lesions with a phase rim $(62 \%)$ also showed a rim on $\mathrm{T}^{*}$ magnitude images (Fig $1)$. The intrarater and interrater agreement results are provided in Table 2. The reliability between each rater and the final consensus agreement was "almost perfect," with a Cohen $\kappa$ ranging from 0.81 to 0.91 and a pair-wise agreement ranging from $91 \%$ to $96 \%$.

\section{Lesion Rim Assessment at 3T versus 7T and Analysis of Disagreements}

Rims were seen in 34 lesions on 7T phase images and in 36 lesions on $3 \mathrm{~T}$ phase images by consensus. The reliability between the consensus agreement at $3 \mathrm{~T}$ versus $7 \mathrm{~T}$ was substantial/good, with a Cohen $\kappa$ of 0.78 and a pair-wise agreement of $90 \%$. After discussion, discrepancies between $3 \mathrm{~T}$ and $7 \mathrm{~T}$ were seen in 10 lesions: 


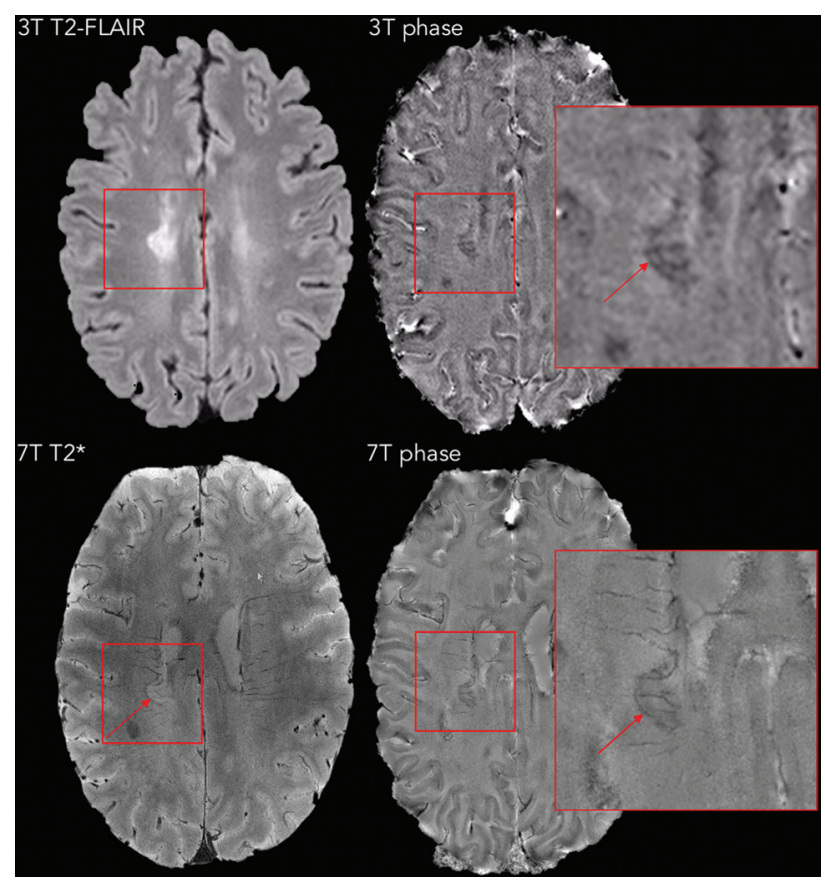

FIG 1. A right periventricular lesion in a 55-year-old woman with relapsing-remitting MS. A paramagnetic rim can be easily seen on $3 \mathrm{~T}$ and 7T phase images (magnified view) as well as on the 7T T2* magnitude image (red arrows). On susceptibility images, medullary veins crossing the lesion are also prominent.

- Four lesions were considered to have been missed at 3T ("falsenegative"). One possible explanation is a small susceptibility effect at the edge of these lesions because none had a $\mathrm{T}^{*}$ rim.

- Three lesions identified as having rims at $3 \mathrm{~T}$ did not have rims at 7T and were therefore considered "false-positive" at 3T. On review, this misinterpretation was thought to be related to a "rim-like" configuration of vessels at the lesion edge.

- Two lesions were considered to have been missed at 7T. On review, this was thought to be due to the relatively thick slices in the $7 \mathrm{~T}$ acquisition ( $1 \mathrm{~mm}$ versus $0.65 \mathrm{~mm}$ at $3 \mathrm{~T}$ ).

- One lesion, even after re-evaluation, was considered to have a rim at $3 \mathrm{~T}$ but not at $7 \mathrm{~T}$. For this lesion, the $3 \mathrm{~T}$ scan preceded the 7T scan by 1 month, and none of the other lesions in this case were discordant.

Representative concordant and discordant cases are shown in Figs $1-3$.

\section{DISCUSSION}

In this study, we tested the reliability of 3T susceptibility-based imaging in visualizing 7T-detected paramagnetic rims in nonenhancing MS lesions. Anticipated difficulties revolved mainly around the lower sensitivity of 3T MR imaging to susceptibility effects, as well as the lower voxel volume of the $3 \mathrm{~T}$ acquisition. At 7T MR imaging, the mean rim thickness in nonenhancing MS lesions has been estimated at $\sim 430 \mu \mathrm{m}$ (range, $150-1200 \mu \mathrm{m}),{ }^{14}$ indicating the necessity of acquiring submillimeter susceptibilitybased imaging. Here, we implemented a 3T whole-brain 3D segmented EPI sequence, providing $0.65-\mathrm{mm}$ isotropic voxels and both $\mathrm{T}^{\star}$ magnitude and phase contrasts. This is the optimized sequence for FLAIR ${ }^{\star}$ imaging $^{16}$ and the related assessment of the central vein sign in MS lesions. ${ }^{17-21}$ The potential of detecting central vein and paramagnetic rims with the same sequence would also be a clear advantage in terms of acquisition time in a clinical setting. In addition, like the central vein, small 7T MR imaging studies showed that lesions with rims are not frequently seen in other inflammatory disorders of the central nervous system such as neuromyelitis optica spectrum disorder ${ }^{25,26}$ and Susac syndrome. ${ }^{27}$

Overall, detection of paramagnetic rims performed well at 3T; representative examples are shown in Figs 1 and 2. Intra- and interrater agreement was substantial/good or better ${ }^{28}$ for both $3 \mathrm{~T}$ and 7T. Most important, agreement was not perfect at 7T; this outcome suggests that a clear MR imaging definition of what constitutes a rim is necessary for future research studies (population analysis) and/or MR imaging-based clinical trials. In addition, our results suggest that dedicated training in MS MR imaging should be a prerequisite for assessing this important morphologic feature at both $3 \mathrm{~T}$ and $7 \mathrm{~T}$.

The discussion that took place within our group during the consensus process highlighted several important observations about imaging of paramagnetic rim lesions at 3T. On the one hand, rim detection on $3 \mathrm{~T}$ phase images was more reliable when the rim was seen: 1) on $\mathrm{T} 2{ }^{\star}$ magnitude and phase images, indicating stronger susceptibility effects; 2 ) on $>1$ consecutive slice; and 3) on multiple orthogonal views. On the other hand, rim detection was less reliable: 1 ) in areas with a high density of veins, such as around the ventricular horns; 2) within the corpus callosum; 3 ) when the rim was seen only partially around the lesion; 4) when the rim did not match the edge of the lesion seen on T2FLAIR images; and 5) in areas with motion and/or bulk susceptibility artifacts.

One important limitation of phase imaging at both 3T and 7T is the presence of dipolar fields surrounding cerebral vessels and some MS lesions. These dipolar projections may hinder the detection of phase rims (especially thin ones) or may be misinterpreted as (false-positive) phase rims. ${ }^{29,30}$ One way to remove these dipolar fields is to apply an extra processing step called Quantitative Susceptibility Mapping (QSM), which computes the magnetic susceptibility of tissues by solving the field-to-source inversion problem using the dipole model. ${ }^{31}$ However, the appearance of QSM images depends heavily on the choice of the algorithm and parameters used for solving the inversion problem. ${ }^{32}$ Moreover, QSM images are typically smoothed. This step can result in a loss of conspicuity for fine structures such as the thin paramagnetic rims frequently observed in our study (On-line Figure). Therefore, further development is warranted for establishing a robust and effective QSM algorithm that can display all types of paramagnetic rims around MS lesions.

Potential limitations of our study are the limited number of lesions included in the analysis and the sometimes long time lapse between $3 \mathrm{~T}$ and $7 \mathrm{~T}$ MR imaging acquisitions, which ranged from 2 days to 3.4 years (mean, 0.9 years, median, 0.7 years). Because paramagnetic rims in chronic lesions have been consistently shown to remain stable for similar durations of follow-up, ${ }^{13-15}$ any detection difference is more likely to be 


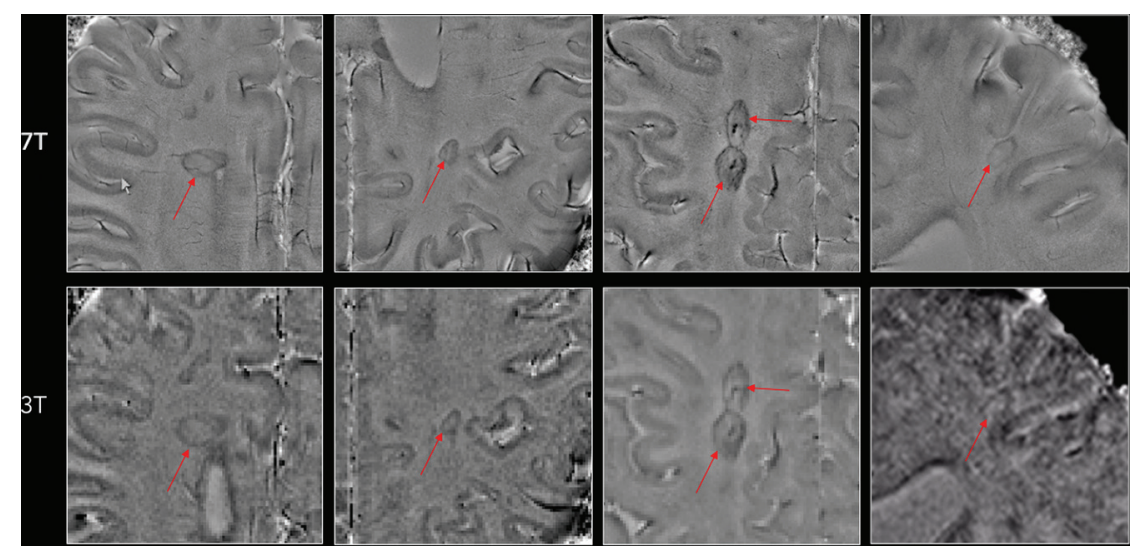

FIG 2. Representative examples of MS lesions in which a paramagnetic rim (red arrows) can be easily seen on both 7T and 3T phase images. Note the superior image quality at 7T in at least 3 of the 4 examples.

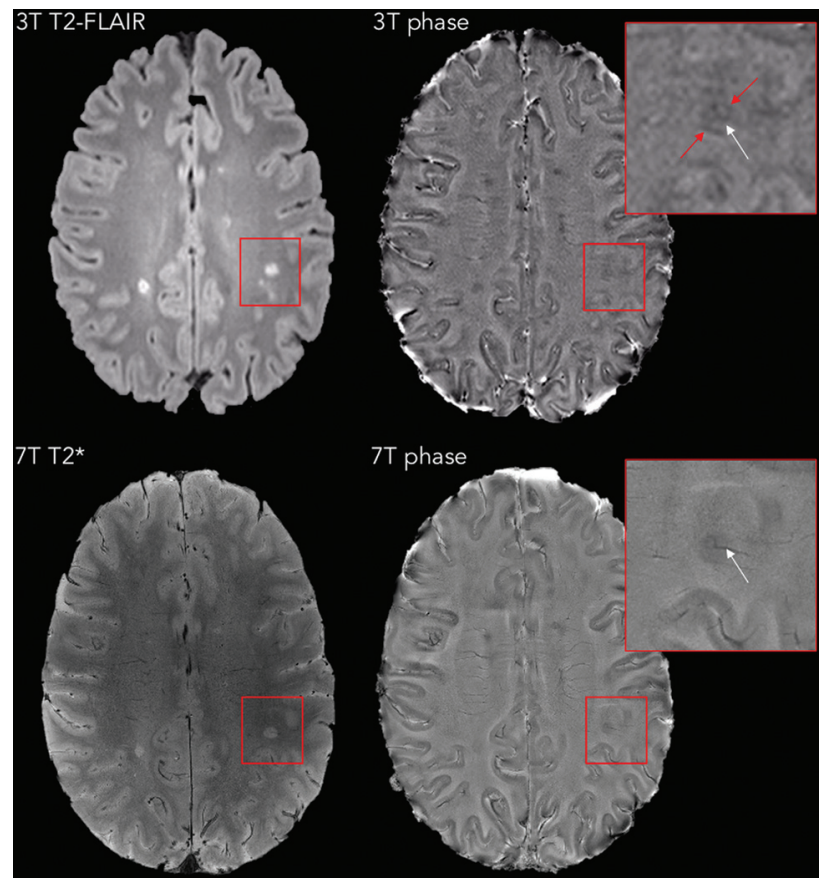

FIG 3. A left centrum semiovale lesion in a 30-year-old woman with relapsing-remitting MS; the time lapse between 3T and 7T scans was 6 months. In this case, the lesion was judged to have a rim on $3 \mathrm{~T}$ (red arrows), but not on 7T phase images. The false-positive rim at 3T is related to the configuration of blood vessels at the lesion edge. On the other hand, the central vein of the lesion is prominent in both phase images (white arrows).

related to different magnetic strengths (3T versus $7 \mathrm{~T}$ ) than to the biologic variability of this feature.

\section{CONCLUSIONS}

Our results show that nearly all 7T paramagnetic rims can also be seen at 3T. This finding suggests the possibility of implementing paramagnetic rims for MS diagnosis and as outcome measures in MR imaging-based clinical trials, potentially affecting perilesional persistent microglial/macrophage-mediated inflammation.

\section{ACKNOWLEDGMENTS}

We thank the study participants, the Neuroimmunology Clinic for recruiting and evaluating the patients and for coordinating the

\section{REFERENCES} Medline scans, and the National Institutes of Health Functional Magnetic Resonance Imaging Facility. We thank Souheil Inati, John A Derbyshire, and Sunil Patil for support with MR imaging sequences and Christian Langkammer for help with QSM image processing.

Disclosures: Martina Absinta—UNRELATED: Grant: National Multiple Sclerosis Society and Conrad N. Hilton Foundation.* Daniel S. Reich—UNRELATED: Grants/Grants Pending: Vertex Pharmaceuticals*; Payment for Lectures Including Service on Speakers Bureaus: At the Limits, Comments: Continuing Medical Education lecture; Travel/Accommodations/ Meeting Expenses Unrelated to Activities Listed: Verily Life Sciences, Biogen, Comments: support for travel and accommodations to present scientific lectures.* *Money paid to the institution.

1. Lassmann H, Raine CS, Antel J, et al. Immunopathology of multiple sclerosis: report on an international meeting held at the Institute of Neurology of the University of Vienna. J Neuroimmunol 1998;86: 213-17 CrossRef Medline

2. Kuhlmann T, Ludwin S, Prat A, et al. An updated histological classification system for multiple sclerosis lesions. Acta Neuropathol 2017;133:13-24 CrossRef Medline

3. Hammond KE, Metcalf $\mathrm{M}$, Carvajal $\mathrm{L}$, et al. Quantitative in vivo magnetic resonance imaging of multiple sclerosis at 7 Tesla with sensitivity to iron. Ann Neurol 2008;64:707-13 CrossRef Medline

4. Pitt D, Boster A, Pei W, et al. Imaging cortical lesions in multiple sclerosis with ultra-high-field magnetic resonance imaging. Arch Neurol 2010;67:812-18 CrossRef Medline

5. Bagnato F, Hametner S, Yao B, et al. Tracking iron in multiple sclerosis: a combined imaging and histopathological study at $7 \mathrm{Te}-$ sla. Brain 2011;134:3602-15 CrossRef Medline

6. Yao B, Bagnato F, Matsuura E, et al. Chronic multiple sclerosis lesions: characterization with high-field-strength MR imaging. $R a$ diology 2012;262:206-15 CrossRef Medline

7. Hagemeier J, Heininen-Brown M, Poloni GU, et al. Iron deposition in multiple sclerosis lesions measured by susceptibility-weighted imaging filtered phase: a case control study. J Magn Reson Imaging 2012;36:73-83 CrossRef Medline

8. Walsh AJ, Lebel RM, Eissa A, et al. Multiple sclerosis: validation of MR imaging for quantification and detection of iron. Radiology 2013;267:531-42 CrossRef Medline

9. Wisnieff C, Ramanan S, Olesik J, et al. Quantitative susceptibility mapping (QSM) of white matter multiple sclerosis lesions: interpreting positive susceptibility and the presence of iron. Magn Reson Med 2015;74:564-70 CrossRef Medline

10. Absinta M, Sati P, Schindler M, et al. Persistent 7-Tesla phase rim predicts poor outcome in new multiple sclerosis patient lesions. J Clin Invest 2016;126:2597-609 CrossRef Medline

11. Dal-Bianco A, Grabner G, Kronnerwetter C, et al. Slow expansion of multiple sclerosis iron rim lesions: pathology and $7 \mathrm{~T}$ magnetic resonance imaging. Acta Neuropathol 2017;133:25-42 CrossRef

12. Popescu BF, Frischer JM, Webb SM, et al. Pathogenic implications of distinct patterns of iron and zinc in chronic MS lesions. Acta Neuropathol 2017;134:45-64 CrossRef Medline

13. Bian W, Harter K, Hammond-Rosenbluth KE, et al. A serial in vivo 7T magnetic resonance phase imaging study of white matter lesions in multiple sclerosis. Mult Scler 2013;19:69-75 CrossRef Medline

14. Absinta M, Sati P, Gaitán MI, et al. Seven-Tesla phase imaging of acute multiple sclerosis lesions: a new window into the inflammatory process. Ann Neurol 2013;74:669-78 CrossRef Medline 
15. Dal-Bianco A, Hametner S, Grabner G, et al. Veins in plaques of multiple sclerosis patients: a longitudinal magnetic resonance imaging study at 7 Tesla. Eur Radiol 2015;25:2913-20 CrossRef Medline

16. Sati P, George IC, Shea CD, et al. FLAIR ${ }^{\star}$ : a combined MR contrast technique for visualizing white matter lesions and parenchymal veins. Radiology 2012;265:926-32 CrossRef Medline

17. Solomon A, Schindler M, Howard DB, et al. "Central vessel sign" on 3T FLAIR* MRI for the differentiation of multiple sclerosis from migraine. Ann Clin Transl Neurol 2015;3:82-87 CrossRef Medline

18. George IC, Sati P, Absinta M, et al. Clinical 3-Tesla FLAIR* MRI improves diagnostic accuracy in multiple sclerosis. Mult Scler 2016; 22:1578-86 CrossRef Medline

19. Sati P, Oh J, Constable RT, et al. The central vein sign and its clinical evaluation for the diagnosis of multiple sclerosis: a consensus statement from the North American Imaging in Multiple Sclerosis Cooperative. Nat Rev Neurol 2016;12:714-22 CrossRef Medline

20. Campion T, Smith RJ, Altmann DR, et al. FLAIR ${ }^{\star}$ to visualize veins in white matter lesions: a new tool for the diagnosis of multiple sclerosis? Eur Radiol 2017;27:4257-63 CrossRef Medline

21. Samaraweera AP, Clarke MA, Whitehead A, et al. The central vein sign in multiple sclerosis lesions is present irrespective of the $\mathrm{T} 2^{*}$ sequence at 3 T. J Neuroimaging 2017;27:114-21 CrossRef Medline

22. Thompson AJ, Banwell BL, Barkhof F, et al. Diagnosis of multiple sclerosis: 2017 revisions of the McDonald criteria. Lancet Neurol 2018;17:162-73 CrossRef Medline

23. Lublin FD, Reingold SC, Cohen JA, et al. Defining the clinical course of multiple sclerosis: the 2013 revisions. Neurology 2014;83:278-86 CrossRef Medline
24. Kurtzke JF. Rating neurologic impairment in multiple sclerosis: an expanded disability status scale (EDSS). Neurology 1983;33:1444-52 CrossRef Medline

25. Sinnecker T, Dörr J, Pfueller CF, et al. Distinct lesion morphology at 7-T MRI differentiates neuromyelitis optica from multiple sclerosis. Neurology 2012;79:708-14 CrossRef Medline

26. Sinnecker T, Schumacher S, Mueller K, et al. MRI phase changes in multiple sclerosis vs neuromyelitis optica lesions at 7T. Neurol Neuroimmunol Neuroinflamm 2016;3:e259 CrossRef Medline

27. Wuerfel J, Sinnecker T, Ringelstein EB, et al. Lesion morphology at 7 Tesla MRI differentiates Susac syndrome from multiple sclerosis. Mult Scler 2012;18:1592-99 CrossRef Medline

28. Landis JR, Koch GG. The measurement of observer agreement for categorical data. Biometrics 1977;33:159-74 CrossRef Medline

29. Eskreis-Winkler S, Deh K, Gupta A, et al. Multiple sclerosis lesion geometry in quantitative susceptibility mapping (QSM) and phase imaging. J Magn Reson Imaging 2015;42:224-29 CrossRef Medline

30. Cronin MJ, Wharton S, Al-Radaideh A, et al. A comparison of phase imaging and quantitative susceptibility mapping in the imaging of multiple sclerosis lesions at ultrahigh field. MAGMA 2016;29: 543-57 CrossRef Medline

31. Deistung A, Schweser F, Reichenbach JR. Overview of quantitative susceptibility mapping. NMR Biomed 2017;30 CrossRef Medline

32. Langkammer C, Schweser F, Shmueli K, et al. Quantitative susceptibility mapping: report from the $\mathbf{2 0 1 6}$ reconstruction challenge. Magn Reson Med 2018;79:1661-73 CrossRef Medline 